\title{
Styloidogenic-cervical spondylotic internal jugular venous compression, a vascular disease related to several clinical neurological manifestations: diagnosis and treatment-a comprehensive literature review
}

\author{
Alba Scerrati ${ }^{1,2} \wedge$, Nicoló Norri ${ }^{1}$, Lorenzo Mongardi ${ }^{1}$, Flavia Dones ${ }^{1}$, Luca Ricciardi ${ }^{3}$, Gianluca Trevisi ${ }^{4}$, \\ Erica Menegatti ${ }^{2,5}$, Paolo Zamboni ${ }^{2,5}$, Michele Alessandro Cavallo ${ }^{1,2}$, Pasquale De Bonis ${ }^{1,2}$ \\ ${ }^{1}$ Neurosurgery Department, Sant'Anna University Hospital, Ferrara, Italy; ${ }^{2}$ Department of Translational Medicine and for Romagna, University \\ of Ferrara, Ferrara, Italy; ${ }^{3}$ Neurosurgery, Azienda Ospedaliera Sant'Andrea, Sapienza, Rome, Italy; ${ }^{4}$ Neurosurgical Unit, Ospedale Santo Spirito, \\ Pescara, Italy; ${ }^{5}$ HUB Center Regione Emilia Romagna for Venous and Lymphatics Disorders, University Hospital of Ferrara, Ferrara, Italy \\ Contributions: (I) Conception and design: A Scerrati, P Zamboni; (II) Administrative support: E Menegatti, L Mongardi; (III) Provision of study \\ materials or patients: N Norri, P De Bonis; (IV) Collection and assembly of data: MA Cavallo, L Ricciardi, G Trevisi; (V) Data analysis and \\ interpretation: F Dones, A Scerrati; (VI) Manuscript writing: All authors; (VII) Final approval of manuscript: All authors. \\ Correspondence to: Dr. Alba Scerrati, MD. Via Aldo Moro 8, Ferrara 44121, Italy. Email: a.scerrati@gmail.com.
}

\begin{abstract}
Internal jugular vein (IJV) stenosis is associated with several central nervous system disorders such as Ménière or Alzheimer's disease. The extrinsic compression between the styloid process and the C1 transverse process, is an emerging biomarker related to several clinical manifestations. However, nowadays a limited number of cases are reported, and few information are available about treatment, outcome and complications. Our aim is to collect and identify clinical-radiological characteristics, diagnosis and treatment of the styloidogenic internal jugular venous compression. We performed a comprehensive literature review. Studies reporting patients suffering from extracranial jugular stenosis were searched. For every patient we collected: demography, clinical and radiological characteristics and outcome, type of treatment, complications. Thirteen articles reporting 149 patients were included. Clinical presentation was nonspecific. Most frequent symptoms were headache (46.3\%), tinnitus (43.6\%), insomnia (39.6\%). The stenosis was monolateral in 51 patients $(45.9 \%)$ and bilateral in 60 (54.1\%). Anticoagulants were the most common prescribed drug (57.4\%). Endovascular treatment was performed in 50 patients $(33.6 \%)$, surgery in 55 (36.9\%), combined in 28 (18.8\%). Improvement of general conditions was reported in 58/80 patients (72.5\%). Complications were reported in $23 \%$ of cases. Jugular stenosis is a complex and often underestimated disease. Conservative medical treatment usually fails while surgical, endovascular or a combined treatment improves general conditions in more than $70 \%$ of patients.
\end{abstract}

Keywords: Eagle jugular syndrome; styloidogenic jugular compression; jugular stenosis; internal jugular vein (IJV); $\mathrm{C} 1$ transverse process; elongated styloid process

Submitted Nov 27, 2020. Accepted for publication Feb 10, 2021.

doi: $10.21037 /$ atm-20-7698

View this article at: http://dx.doi.org/10.21037/atm-20-7698

\footnotetext{
$\wedge$ ORCID: 0000-0002-8607-5323.
} 


\section{Introduction}

Several central nervous system disorders, such as Ménière disease, idiopathic intracranial hypertension, multiple sclerosis, transient monocular blindness and Alzheimer's disease have been reported to be associated with internal jugular vein (IJV) stenosis (1-5). The stenosis can be caused by thrombosis, wall issues or various extrinsic compressions (6-8).

In particular, IJV extrinsic compression at the passage between the $\mathrm{C} 1$ transverse process and the styloid process, is an emerging nosological entity that can be related to several clinical manifestations (9-13).

It presents with anatomical features of Eagle syndrome (an elongated styloid process impinging the surrounding blood vessels) and has been associated with intracranial hypertension and venous outflow reduction (11). In the jugular variant the impingement is usually between the styloid process and the arch of $\mathrm{C} 1$. The elongation of the styloid process is not the only causative factor, but the process itself could present a specific orientation causing an impression on the jugular vein.

Clinical presentation may be variable and nonspecific (headache, anxiety and neck discomfort, head noises, tinnitus, hearing loss, memory loss, visual disorders, insomnia) (14), and due to the limited number of cases reported in literature, no clear definitions or classifications are nowadays available. Several treatments have been proposed including diuretics, surgery or endovascular procedures.

Aim of our study is to perform a comprehensive literature review in order to identify the clinical characteristics of the styloidogenic-cervical spondylotic internal jugular venous compression (SJVC), to better define its clinical characteristics, diagnosis and treatment.

\section{Materials and methods}

We performed a literature review, following the preferred reporting items for systematic review and meta-analysis (PRISMA) statement. The review question was formulated according to the PICO criteria, as follows: ( $\mathrm{P}$ - patients) in patients with styloidogenic jugular compression, (Iintervention) what kind of treatment are proposed $(\mathrm{C}-$ comparison) and what are the results, (O-outcomes) in terms of clinical-radiological outcomes?

\section{Study selection}

Common medical databases such as PubMed, Ovid Medline, Ovid EMBASE, Scopus, and Web of Science were used to conduct this systematic review. The search terms were "jugular", "stenosis", "extracranial", "C1 transverse process", "styloid process", "styloidogenic compression", "spondylotic compression", "eagle", "cervical”, [MeSH], combined using the Boolean operators.

Studies reporting data on patients suffering from extracranial jugular stenosis were searched. Inclusion criteria: papers written in English language, availability of clinical and radiological data for single patients. We excluded guidelines, commentaries, letters to the editor.

Two reviewers ( $\mathrm{LM}$ and $\mathrm{NN}$ ) conducted the first-round search and independently screened Titles and Abstracts for eligibility. They selected full-texts and performed a forward search for their references list including further papers in the second round.

Afterwards, papers were screened for, jugular site stenosis, demographics, clinical status, treatment modalities, clinical and radiological outcomes, procedural-related complications. The paper was then excluded in case of improper data reporting (exclusion with reason). In the lastround data were collected and databased for pooling and the numerical analysis (inclusion). Discordances were discussed and solved by consensus with the senior author (AS).

\section{Outcomes measurement}

We collected the following data for every patient: demography, clinical (headache, visual impairment, papilledema, soft tissue swelling, neck pain or discomfort, tinnitus, dizziness, ICP hypertension, nausea and vomiting, insomnia, hearing impairment) and radiological (monolateral or bilateral stenosis, stenotic segment, styloid process elongation, arch of C1 hypertrophy, collateral venous circulation, intracranial venous thrombosis, type of imaging diagnostics) characteristics, type of treatment (medical conservative, surgery or endovascular), clinical and radiological outcome, perioperative complications. Clinical outcome was scored as resolved, improved or unchanged.

\section{Results}

Following the search strategy, 16 articles in English 

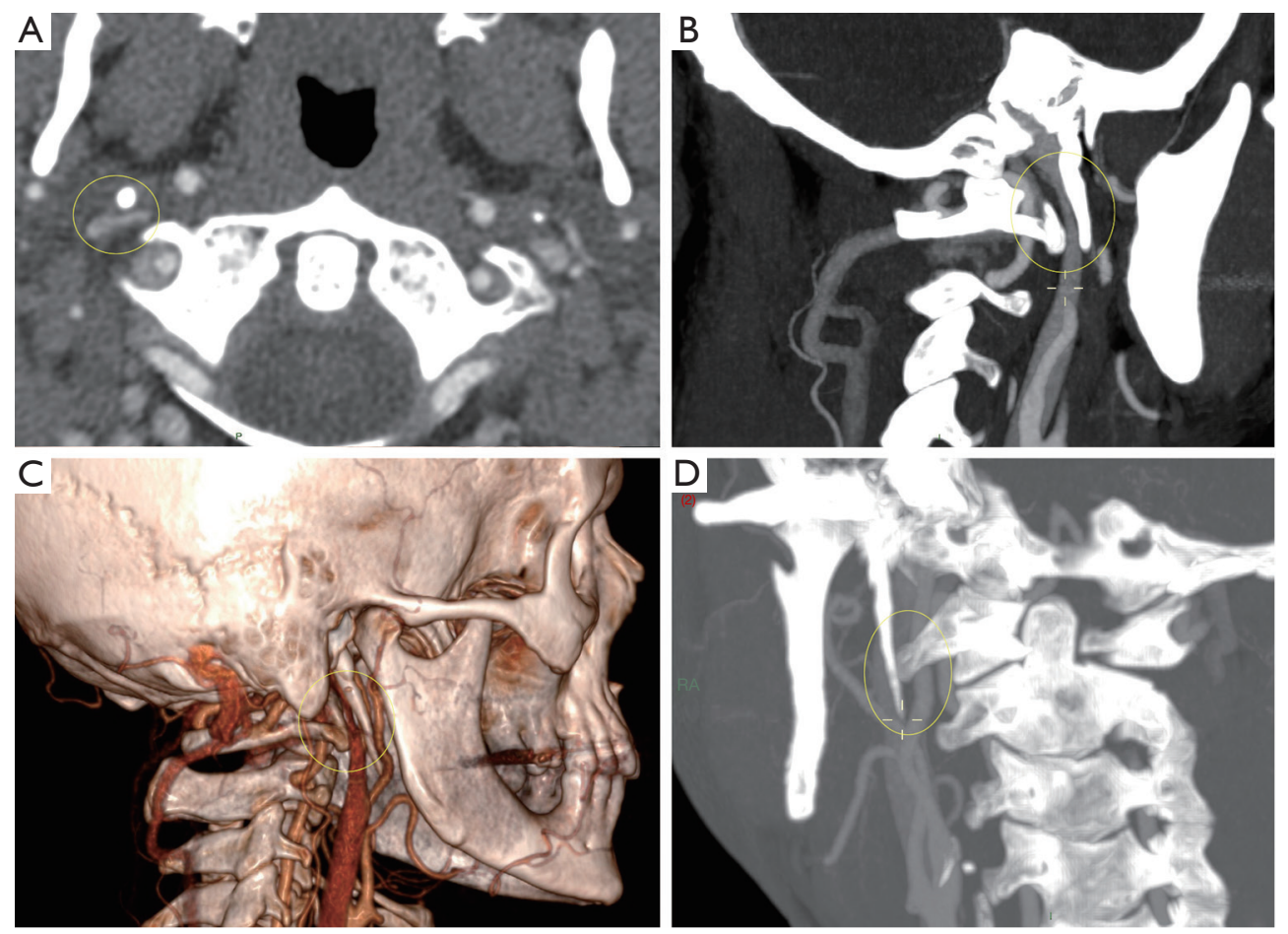

Figure 1 A patient suffering from the Eagle Jugular Syndrome. The internal jugular vein is impinged between the styloid process and the arch of C1 (yellow circle). (A) CT angio axial cut; (B) CT angio sagittal cut; (C) 3D reconstruction of the CT angio; (D) Oblique view.

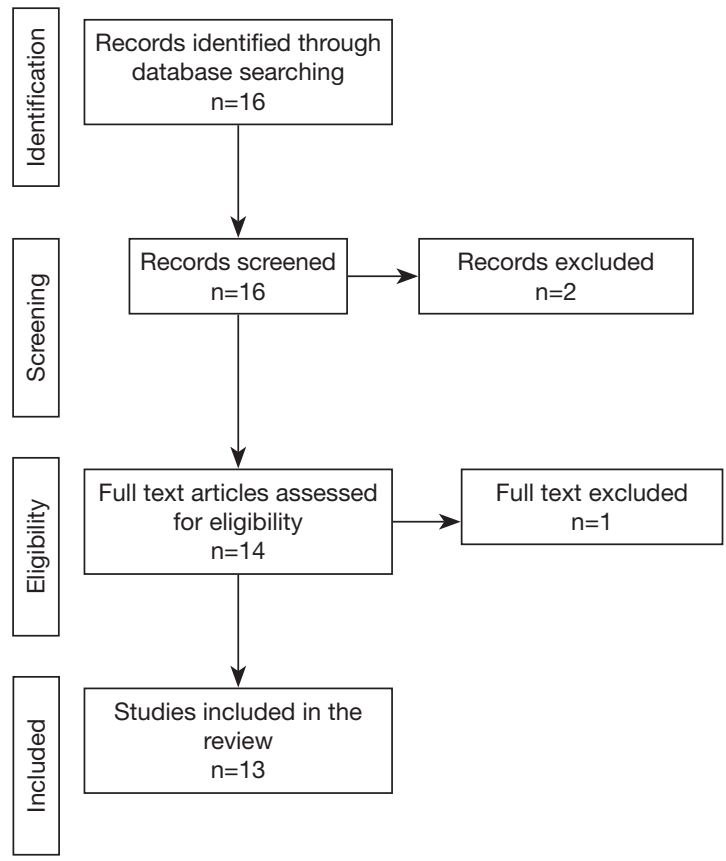

Figure 2 Flow chart of the data selection. language were retrieved through the electronic literature search.

Two papers were primarily excluded after abstract reading, while 14 (meeting our inclusion criteria) were considered for eligibility and analyzed in detail.

After full-text reading and a forward search from the bibliography of the selected papers, 13 articles (9-11,13-22) (12 retrospectives and 1 prospective) published between 2012 and 2020 reporting 149 patients suffering from SJVC, were finally included in this review (Figure 1).

We launched the last search in October 2020.

The flow chart of the data selection is reported in Figure 2.

\section{Demographic characteristics}

We collected 149 patients, 75 males (50.3\%) and 74 females (49.7\%). Mean age was 38.6 years (standard deviation was reported in a minority of series). 
Table 1 Patients' clinical characteristics $(\mathrm{N}=149)$

\begin{tabular}{lc}
\hline Clinical symptoms & $\mathrm{n}(\%)$ \\
\hline Headache & $69(46.3)$ \\
Visual impairment & $43(28.9)$ \\
Papilledema/optic nerve atrophy & $19(12.6)$ \\
Visual impairment & $13(8.7)$ \\
Soft tissue swelling & $1(0.7)$ \\
Neck pain or discomfort & $22(14.8)$ \\
Tinnitus & $65(43.6)$ \\
Dizziness & $33(22.1)$ \\
ICP hypertension & $54(36.2)$ \\
Nausea and vomiting & $6(4.0)$ \\
Insomnia & $55(39.6)$ \\
Hearing impairment & $36(24.2)$ \\
\hline
\end{tabular}

ICP, increased intracranial pressure.

Table 2 Patients' radiological characteristics

\begin{tabular}{lc}
\hline Radiological findings & No. of patients (\%) \\
\hline Monolateral stenosis & $51 / 111(45.9)$ \\
Bilateral stenosis & $60 / 111(54.1)$ \\
Jugular stenotic segment & \\
J1 & $4 / 136(2.9)$ \\
J2 & $8 / 136(5.9)$ \\
J3 & $124 / 136(91.2)$ \\
Styloid process elongation & $48 / 149(32.2)$ \\
Arch of C1 hypertrophy & $36 / 149(24.2)$ \\
Collateral venous circulation & $28 / 149(18.8)$ \\
Intracranial venous thrombosis & $9 / 149(6.0)$ \\
Venous DSA & \\
No & $13 / 149(8.7)$ \\
Venography & $83 / 149(55.7)$ \\
Manometry & $28 / 149(18.8)$ \\
Both & $25 / 149(16.8)$ \\
\hline
\end{tabular}

DSA, digital subtraction angiography.

\section{Clinical characteristics}

Clinical presentation was very non-specific. Most frequent symptoms were headache (46.3\%), tinnitus (43.6\%), insomnia (39.6\%) and visual disturbances $(28.9 \%)$. Increased intracranial pressure (ICP) was reported in $36.2 \%$ of patients.

See Table 1 for further details.

\section{Radiological characteristics}

The stenosis was monolateral in 51 out of 111 patients (45.9\%) and bilateral in 60 (54.1\%) (Figure 1). The most frequent stenotic segment was J3 (91.2\%), the styloid process elongation was reported in $32.2 \%$ of patients, while the $\mathrm{C} 1$ arch hypertrophy in $24.2 \%$ of patients. All patients underwent CT or MR angio and arterial DSA, while 83 patients $(55.7 \%)$ underwent venography, 28 (18.8\%) manometry and 25 (16.8\%) both. See Table 2 for further details.

\section{Type of treatment}

All patients were medically treated, and anticoagulants were the most common prescribed drug (57.4\%). Other treatments were statins, antithrombotics or xueshuantong.

Endovascular treatment was performed in 50 out of 149 patients $(33.6 \%)$ and stenting was performed in 45 of them.

Surgery was performed in 55 out of 149 patients (36.9\%) and consisted of: styloidectomy in 41 patients $(27.5 \%), \mathrm{C} 1$ tubercolectomy in 2 patients (1.3\%), styloidectomy and $\mathrm{C} 1$ tubercolectomy in 12 patients $(8.1 \%)$.

A combined treatment (styloidectomy and stenting) was reported in 28 out of 149 patients (18.8\%).

Further details are reported in Table 3.

\section{Clinical and Radiological outcome}

Improvement of general conditions was reported in 58 out of 80 patients $(72.5 \%)$. In detail, tinnitus resolved or improved in 13 out of 15 patients (86.7\%), papilledema in 17 out of $22(77.2 \%)$ patients and visual disturbances 
Table 3 Patients' treatment characteristics

\begin{tabular}{llc}
\hline Type of treatment & Drug/procedures & No. of patients (\%) \\
\hline Medical treatment & Acetazolamide & $17 / 141(12.1)$ \\
& Anticoagulants & $81 / 141(57.4)$ \\
& Others & $43 / 141(30.5)$ \\
Endovascular treatment & Balloon angioplasty & $4 / 149(2.7)$ \\
& Balloon angioplasty + stent & $1 / 149(0.7)$ \\
Surgery & Stent & $45 / 149(30.2)$ \\
& Styloidectomy & $41 / 149(27.5)$ \\
Combined & C1 tubercolectomy & $2 / 149(1.3)$ \\
\hline
\end{tabular}

improved in 10 out of 23 patients (43.5\%). Poor improvements were reported for headache and dizziness, which remained unchanged for $71.6 \%$ and $90.9 \%$ of patients respectively (see Table 4).

Stenosis was radiologically improved in 45 out of 63 surgically treated patients $(71.4 \%)$, while was improved in 16 out of 63 patients who underwent endovascular treatment $(25.4 \%)$.

Complications were reported in 9 out of 39 cases (23\%): 1 wound infection, 1 wound hematoma, 3 transient neurological deficits $<24 \mathrm{~h}, 1$ permanent neurological deficit (accessory nerve palsy), 1 facial droop and dysphagia, 2 numbness of jaw, neck and ear.

List of authors, year of publication, study type and number of patients of the published studies included in the review are summarized in Table 5 .

\section{Discussion}

Extra-cranial IJV compression is not a rare finding in routine clinical practice (23) and it is difficult to refer to particular symptoms or syndromes. Jayaraman et al. (6) published a study, enrolling 108 patients which underwent CT angios. The incidence of IJV compression by extrinsic structures in the upper neck was investigated. They considered jugular vein compression as an anatomic variant and unlikely to be pathologic in nature. Indeed, no data about the reasons why these CT angio were performed or patient's clinical history were reported. In our opinion, it can be considered only a radiological report with a lack of clinical correlation.
However, styloidogenic jugular compression is an increasingly described new nosological entity, named with different titles, such as "styloidogenic-cervical spondylotic internal jugular venous compression”, "eagle jugular syndrome" or "styloid-induced internal jugular vein stenosis". The lack of awareness of this disease entity could lead to misdiagnose and treatment delay.

Aim of our review was to collect all clinical and radiological data about this disease in order to better define its characteristics and provide reference for its diagnosis and treatment.

There is no prevalence between female or male population and the mean age of onset is 38.6 years. The underlying etiopathological mechanism is still not clear and is more frequently being discussed in the scientific community. The clinical presentation seems to be mostly characterized by headache (46.3\%), tinnitus $(43.6 \%)$ and visual disturbances (28.9\%). Surprisingly, insomnia, a very aspecific and often underestimated symptom, affects nearly $40 \%$ of patients. These symptoms could be partially ascribed to an increased ICP, which was measured and reported in $36.2 \%$ of patients.

In addition to tinnitus, hearing impairment or dizziness were reported in about $1 / 4$ of patients, suggesting also blood flow circulatory disturbances in auditory-related structures (14).

The rationale to hypothesize a possible pathological potential of styloidogenic jugular compression lies in the following considerations.

In the Gadda-Ursino hemodynamic model for the study of cerebral venous outflow, a jugular stenosis may have a 
Table 4 Final clinical and radiological outcomes

\begin{tabular}{|c|c|c|c|}
\hline Outcome & & & No. of patients \\
\hline \multirow{12}{*}{ Clinical outcome } & General conditions & Unchanged & $22 / 80(27.5)$ \\
\hline & \multirow[t]{2}{*}{ Visual disturbance } & Improved & $10 / 23(43.5)$ \\
\hline & & Unchanged & $13 / 23(56.5)$ \\
\hline & \multirow{2}{*}{ Papilledema } & Improved & $12 / 22(54.5)$ \\
\hline & & Unchanged & 5/22 (22.7) \\
\hline & \multirow[t]{2}{*}{ Headache } & Resolved & 19/88 (21.6) \\
\hline & & Improved & $6 / 88(6.8)$ \\
\hline & \multirow{2}{*}{ Dizziness } & Improved & $2 / 33(6.1)$ \\
\hline & & Unchanged & $30 / 33(90.9)$ \\
\hline & \multirow[t]{3}{*}{ Tinnitus } & Resolved & $10 / 15(66.7)$ \\
\hline & & Improved & 3/15 (20.0) \\
\hline & & Unchanged & 2/15 (13.3) \\
\hline \multirow[t]{3}{*}{ Radiological outcome } & \multirow[t]{3}{*}{ Stenosis improvement } & After surgery & 45/63 (71.4) \\
\hline & & After endovascular treatment & $16 / 63(25.4)$ \\
\hline & & After combined treatment & 2/63 (3.2) \\
\hline
\end{tabular}

strong effect on venous sinuses pressure (24). These findings were clinically confirmed by Esfahani with quantitative magnetic resonance venography (qMRV) (25). The venous pressure is strongly correlated to cerebrospinal fluid (CSF) dynamics (26). A computational study investigated the effect of internal-jugular vein function on intracranial venous haemodynamics, in particular focusing on venous reflux and intracranial venous hypertension. This study concluded that the clinical implications of the findings are unknown, though they may relate to recent hypotheses linking some neurological conditions to extra-cranial venous anomalies (27).

A venous flow impairment, even if monolateral, due to the styloidogenic compression could bring an imbalance in this sensitive venous-CSF mechanism, resulting in causing symptoms (headache, tinnitus, insomnia, visual impairment etc.) not unexplainable otherwise with common neurosurgical/ENT pathologies. In addition, the venous system itself can be considered as a pure mechanical parallel system. However, the relationship and the balance with the CSF dynamics is a more complex system. In 2012 a group of researchers hypothesized the existence of the "glymphatic system". According to them, CSF quickly enters the brain, along the paravascular spaces of the penetrating arteries, then exchanges with the surrounding interstitial fluid. Likewise, interstitial fluid is dismissed from the brain parenchyma through paravascular spaces surrounding large draining veins (28).

Impairment in venous drainage reflects on CSF dynamics that could be responsible of several symptoms associated to styloidogenic jugular vein compression (headache, tinnitus, visual disturbances) (29-31).

Probably IJV stenosis clinical manifestations may originate by more complex pathological mechanisms, most of them still unknown, rather than only by a mechanical compression with subsequent venous flow reduction.

The diagnosis still remains radiological, with the 
Table 5 List of authors, year of publication, study type of number of patients of the published studies included in the review

\begin{tabular}{lccc}
\hline First author & Year & Study type & No. of patients \\
\hline Dashti et al. & 2011 & Case series & 2 \\
Higgins et al. & 2016 & Retrospective review & 29 \\
Aydin et al. & 2016 & Case series & 2 \\
Zhang et al. & 2017 & Case series & 15 \\
Bai et al. & 2019 & Case series & 27 \\
Ding et al. & 2019 & Prospective study & 46 \\
Li et al. & 2019 & Case report & 1 \\
Li et al. & 2019 & Case series & 5 \\
Zamboni et al. & 2019 & Case series & 8 \\
Zhao et al. & 2019 & Retrospective review & 10 \\
Mooney et al. & 2020 & Case report & 1 \\
Fritch et al. & 2020 & Case report & 1 \\
Pokeerbux et al. & 2020 & Case report & \\
\hline
\end{tabular}

evidence of extracranial jugular stenosis usually due to an elongated styloid process or the arch of C1 hypertrophy (Figure 1).

The most common involved jugular segment is $\mathrm{J} 3$, and in more than $50 \%$ of patients the stenosis is bilateral.

Interestingly, CT or MR angio were performed in all patients, however a DSA venography was performed in about $50 \%$ of patients, and only $19 \%$ of them underwent a manometry, resulting in poor data about the venous flow and pressure.

The styloid process elongation was reported in only $1 / 3$ of patients. This confirmed data by Ho et al. that even in the setting of normal length styloid process, a narrowed distance between the styloid process and C1 transverse process may lead to IJV compression (32).

The first choice of treatment is usually conservative, and anticoagulation was the most common reported approach $(57 \%)$. Diuretics, such as acetazolamide, were limited to few cases; while other types of drugs, like statins or antithrombotics, were administered in $1 / 3$ of patients. However, in most cases medical therapy has shown to be not effective.

Forty-five $(30.2 \%)$ patients underwent a stenting procedure and in 28 cases it was associated with surgery. Despite styloidectomy is the treatment of choice in most cases of classic Eagle syndrome or involving carotid arteries, data about cases of symptomatic IJV compression are limited (32). Indeed, often in order to obtain a good jugular flow restoring, endovascular treatment and surgery need to be combined: on one hand the endovascular procedure alone could not be effective if the mechanical compression by the bony structures (styloid process or arch of C1) is not relieved (15); on the other hand surgery alone could not be sufficient to completely re-expand the chronic compressed vein, because of the venous wall structure itself lacking of muscular layers; because of this, stent placement allows to mechanically restore the vessel diameter (9).

Styloidectomy was the most common surgical procedure and was performed as primary or rescue treatment (15). Major risks are represented by vascular or facial nerve injuries $(10,20,33)$.

As alternative, the resection of the arch of $\mathrm{C} 1$ has been proposed (17) especially when deep indentation of the lateral mass is seen exactly at the point of stenosis during the computed tomography $(33,34)$.

In terms of clinical outcome, difficulties were found in the interpretation of reported data, which were generic, available in a small number of patients (from 15 to 80 , depending on the outcome) and with a short time of followup (maximum 14 months).

Beyond that, general conditions were improved in more than $70 \%$ of patients; tinnitus, papilledema and visual disturbances seem to benefit from operative treatments, while headache and dizziness usually do not respond (see 
Table 4).

Radiological outcome was reported in 63 patients out of 149 . Surgery alone seems to guarantee better results (71.4\% of stenosis improvement). Unfortunately, the larger study (15) describing a combined treatment (styloidectomy and stenting), does not report the radiological outcome.

Complications were reported in 9 out of 39 patients $(23 \%)$ and mostly correlated to surgical procedures.

Although complications are rarely reported and discussed in the included papers, these must never be underestimated. Endovascular venous stenting has been associated to stent migration or fracture, pseudoaneurysms formation, stent thrombosis, cerebral sino-venous thrombosis, cranial nerve injury and injury associated with venous catheterization (35).

Surgical complications are represented by wound infection, postoperative hematoma, facial weakness, dysphasia, accessory nerve or facial nerve injury (15).

While some complications can be asymptomatic (like vein thrombosis and stent migration), these future risks including the potential need for long-term anticoagulation and the possibility of stent thrombosis need to be seriously considered. When performing this kind of procedures, physicians should be aware of potential risks, and continue to develop strategies to monitor and manage these patients.

Evidence nowadays available in the literature does not allow to support any kind of robust conclusion but hints at the imperative to collect more data about this pathology and its treatment. The surgical or endovascular treatments should be considered after failure of conservative treatment and need to be carefully evaluated and validated with stronger evidence (RCTs) in order to really understand beneficial effects and possible complications. Our results only suggest these kinds of treatment could have a role in the treatment of styloidogenic jugular compression.

Styloidogenic jugular compression should be considered as a complex pathological entity, which deserves more attention due to the limited current available information and the long-lasting neurological symptoms not attributable to other known diseases.

\section{Open issues and future perspectives}

The complete pathogenesis of SJVC is still not clear. A color doppler ultrasound of jugular vein is a non-invasive diagnostic exam which could allow us to collect information about the flow velocity, flow rate and diversion of flow through the collaterals normalized to the individual inflow $(36,37)$. The same parameters can be non- invasively collected by the means of cervical strain-gauge plethysmography (38). Both can be very useful tools, either for the screening or for the follow-up of patients suffering from this condition.

Indeed, more invasive exams such as DSV (digital subtraction venography) and manometry should also be performed in all patients in order to collect adjunctive physio-pathological parameters.

One of the major issues of styloidogenic jugular compression is the lack of a standardized definition. We carefully analyzed definitions and methodologies reported in the 13 papers we included in the review. Indeed, most of the papers are case reports and refer generally to "elongated styloid process", or "jugular stenosis" or "compression from the transverse process of $\mathrm{C} 1$ combined with the styloid process". Only 2 papers by Ding et al. and Bai et al. reported clear and defined criteria to define styloidogenic jugular compression $(14,19)$. Nevertheless, for future studies on this topic we should advice the use of a clear definition of jugular stenosis such as a reduction of vessel caliber $>80 \%$, based on diameter reduction on axial cuts compared with diameter of normal vein proximal to the stenosis [according to Jayaraman criteria (6)] or the use of criteria described by Ding and Bai $(14,19)$.

\section{Limits}

Most of the studies were case reports or case series, with a poor quality of evidence.

Moreover, timing, specific type and dosage of medical treatments were not reported in most of the studies.

Clinical and radiological outcomes were reported in a small number of patients. Lack of manometry data is very concerning and may constitute a bias in the results evaluation.

Long term follow up is missing for most of the cases.

Unfortunately, statistics was not applied, because of the heterogeneity of the data. Indeed, we tried to obtain individual data from each selected study, however in several studies this was not possible, and we collected only overall data of patients

\section{Conclusions}

Eagle jugular syndrome is a very complex and nowadays often underestimated disease. The clinical presentation is mainly characterized by headache, tinnitus and visual disturbances. Conservative medical treatment usually fails 
while surgical, endovascular or a combined treatment improves general conditions in more than $70 \%$ of patients.

\section{Acknowledgments}

Funding: None.

\section{Footnote}

Peer Review File: Available at http://dx.doi.org/10.21037/ atm-20-7698

Conflicts of Interest: All authors have completed the ICMJE uniform disclosure form (available at http://dx.doi. org/10.21037/atm-20-7698). The authors have no conflicts of interest to declare.

Ethical Statement: The authors are accountable for all aspects of the work in ensuring that questions related to the accuracy or integrity of any part of the work are appropriately investigated and resolved.

Open Access Statement: This is an Open Access article distributed in accordance with the Creative Commons Attribution-NonCommercial-NoDerivs 4.0 International License (CC BY-NC-ND 4.0), which permits the noncommercial replication and distribution of the article with the strict proviso that no changes or edits are made and the original work is properly cited (including links to both the formal publication through the relevant DOI and the license). See: https://creativecommons.org/licenses/by-nc-nd/4.0/.

\section{References}

1. Chung CP, Hsu HY, Chao AC, et al. Transient global amnesia: cerebral venous outflow impairment-insight from the abnormal flow patterns of the internal jugular vein. Ultrasound Med Biol 2007;33:1727-35.

2. Hsu HY, Chao AC, Chen YY, et al. Reflux of jugular and retrobulbar venous flow in transient monocular blindness. Ann Neurol 2008;63:247-53.

3. Nedelmann M, Kaps M, Mueller-Forell W. Venous obstruction and jugular valve insufficiency in idiopathic intracranial hypertension. J Neurol 2009;256:964-9.

4. Chung CP, Beggs C, Wang PN, et al. Jugular venous reflux and white matter abnormalities in Alzheimer's disease: a pilot study. J Alzheimers Dis 2014;39:601-9.

5. Zamboni P, Galeotti R, Salvi F, et al. Effects of Venous
Angioplasty on Cerebral Lesions in Multiple Sclerosis: Expanded Analysis of the Brave Dreams Double-Blind, Sham-Controlled Randomized Trial. J Endovasc Ther 2020;27:1526602819890110.

6. Jayaraman MV, Boxerman JL, Davis LM, et al. Incidence of extrinsic compression of the internal jugular vein in unselected patients undergoing CT angiography. AJNR Am J Neuroradiol 2012;33:1247-50.

7. Gianesini S, Menegatti E, Mascoli F, et al. The omohyoid muscle entrapment of the internal jugular vein. A still unclear pathogenetic mechanism. Phlebology 2014;29:632-5.

8. Brinjikji W, Graffeo CS, Perry A, et al. Moving target: transient rotational stenosis precipitating jugular bow hunter's syndrome. J Neurointerv Surg 2017;9:e28.

9. Zamboni P, Scerrati A, Menegatti E, et al. The eagle jugular syndrome. BMC Neurol 2019;19:333.

10. Dashti SR, Nakaji P, Hu YC, et al. Styloidogenic jugular venous compression syndrome: diagnosis and treatment: case report. Neurosurgery 2012;70:E795-9.

11. Zhao X, Cavallo C, Hlubek RJ, et al. Styloidogenic Jugular Venous Compression Syndrome: Clinical Features and Case Series. Oper Neurosurg (Hagerstown) 2019;17:554-61.

12. Zhang FL, Zhou HW, Guo ZN, et al. Eagle Syndrome as a Cause of Cerebral Venous Sinus Thrombosis. Can J Neurol Sci 2019;46:344-5.

13. Li M, Sun Y, Chan CC, et al. Internal jugular vein stenosis associated with elongated styloid process: five case reports and literature review. BMC Neurol 2019;19:112.

14. Bai C, Wang Z, Guan J, et al. Clinical characteristics and neuroimaging findings in eagle syndrome induced internal jugular vein stenosis. Ann Transl Med 2020;8:97.

15. Higgins JN, Garnett MR, Pickard JD, et al. An Evaluation of Styloidectomy as an Adjunct or Alternative to Jugular Stenting in Idiopathic Intracranial Hypertension and Disturbances of Cranial Venous Outflow. J Neurol Surg B Skull Base 2017;78:158-63.

16. Mooney J, Lepard J, Akbari SHA, et al. Styloidogenic jugular venous compression syndrome: a case report and review of the literature. Childs Nerv Syst 2020;36:3135-9.

17. Fritch C, Voronovich Z, Carlson AP. C1 Transverse Process Resection for Management of Jugular Stenosis. Oper Neurosurg (Hagerstown) 2020;19:E209-E213.

18. Pokeerbux MR, Delmaire C, Morell-Dubois S, et al. Styloidogenic compression of the internal jugular vein, a new venous entrapment syndrome? Vasc Med 2020;25:378-80. 
19. Ding JY, Zhou D, Pan LQ, et al. Cervical spondylotic internal jugular venous compression syndrome. CNS Neurosci Ther 2020;26:47-54.

20. Li M, Gao X, Rajah GB, et al. Styloidectomy and Venous Stenting for Treatment of Styloid-Induced Internal Jugular Vein Stenosis: A Case Report and Literature Review. World Neurosurg 2019;130:129-32.

21. Aydin E, Quliyev H, Cinar C, et al. Eagle Syndrome Presenting with Neurological Symptoms. Turk Neurosurg 2018;28:219-25.

22. Zhou D, Meng R, Zhang X, et al. Intracranial hypertension induced by internal jugular vein stenosis can be resolved by stenting. Eur J Neurol 2018;25:365-e13.

23. Diehn FE, Schwartz KM, Hunt CH, et al. Prevalence of incidental narrowing of the superior segment of the internal jugular vein in patients without multiple sclerosis. Clin Neuroradiol 2014;24:121-7.

24. Gadda G, Taibi A, Sisini F, et al. A new hemodynamic model for the study of cerebral venous outflow. Am J Physiol Heart Circ Physiol 2015;308:H217-31.

25. Esfahani DR, Stevenson M, Moss HE, et al. Quantitative Magnetic Resonance Venography is Correlated With Intravenous Pressures Before and After Venous Sinus Stenting: Implications for Treatment and Monitoring. Neurosurgery 2015;77:254-60.

26. DE Simone R, Ranieri A, Bonavita V. Starling resistors, autoregulation of cerebral perfusion and the pathogenesis of idiopathic intracranial hypertension. Panminerva Med 2017;59:76-89.

27. Toro EF, Muller LO, Cristini M, et al. Impact of Jugular Vein Valve Function on Cerebral Venous Haemodynamics. Curr Neurovasc Res 2015;12:384-97.

28. Iliff JJ, Wang M, Liao Y, et al. A paravascular pathway facilitates CSF flow through the brain parenchyma and the clearance of interstitial solutes, including amyloid beta. Sci Transl Med 2012;15;4:147ra111.

Cite this article as: Scerrati A, Norri N, Mongardi L, Dones F, Ricciardi L, Trevisi G, Menegatti E, Zamboni P, Cavallo MA, De Bonis P. Styloidogenic-cervical spondylotic internal jugular venous compression, a vascular disease related to several clinical neurological manifestations: diagnosis and treatment-a comprehensive literature review. Ann Transl Med 2021;9(8):718. doi: 10.21037/atm-20-7698
29. Guo P, Sun W, Shi S, et al. Patients with pulsesynchronous tinnitus should be suspected to have elevated cerebrospinal fluid pressure. J Int Med Res 2019;47:4104-13.

30. Grech O, Mollan SP, Wakerley BR, et al. Emerging themes in idiopathic intracranial hypertension. J Neurol 2020;267:3776-84.

31. Atsumi H, Horie T, Kajihara N, et al. Simple Identification of Cerebrospinal Fluid Turbulent Motion Using a Dynamic Improved Motion-sensitized Driven-equilibrium Steady-state Free Precession Method Applied to Various Types of Cerebrospinal Fluid Motion Disturbance. Neurol Med Chir (Tokyo) 2020;60:30-6.

32. Ho S, Luginbuhl A, Finden S, et al. Styloid/C1 transverse process juxtaposition as a cause of Eagle's syndrome. Head Neck 2015;37:E153-6.

33. Scerrati A, De Bonis P, Zamboni P. Letter to the Editor Regarding "Styloidectomy and Venous Stenting for Treatment of Styloid-Induced Internal Jugular Vein Stenosis: A Case Report and Literature Review". World Neurosurg 2020;139:697.

34. Carlson AP, Fritch C. In Reply: C1 Transverse Process Resection for Management of Jugular Stenosis. Oper Neurosurg (Hagerstown) 2020;19:E466.

35. Burton JM, Alikhani K, Goyal M, et al. Complications in MS patients after CCSVI procedures abroad (Calgary, AB). Can J Neurol Sci 2011;38:741-6.

36. Zamboni P, Sisini F, Menegatti E, et al. An ultrasound model to calculate the brain blood outflow through collateral vessels: a pilot study. BMC Neurol 2013;13:81.

37. Zamboni P, Menegatti E, Pomidori L, et al. Does thoracic pump influence the cerebral venous return? J Appl Physiol (1985) 2012;112:904-10.

38. Zamboni P, Menegatti E, Conforti P, et al. Assessment of cerebral venous return by a novel plethysmography method. J Vasc Surg 2012;56:677-85.e1. 